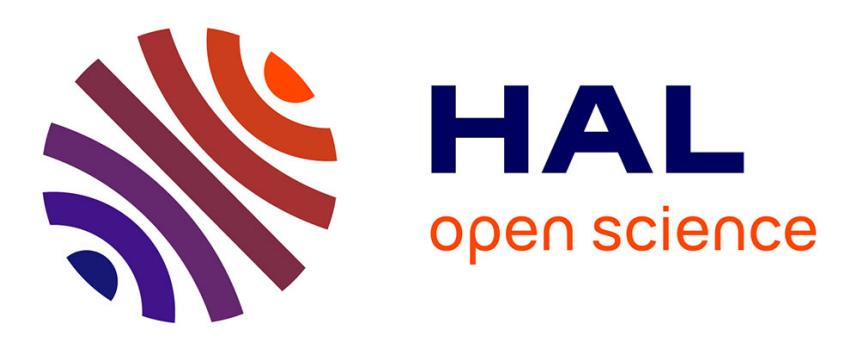

\title{
Agent-Based Security Constrained Optimal Power Flow with Primary Frequency Control
}

Maxime Velay, Meritxell Vinyals, Yvon Besanger, Nicolas Retière

\section{To cite this version:}

Maxime Velay, Meritxell Vinyals, Yvon Besanger, Nicolas Retière. Agent-Based Security Constrained Optimal Power Flow with Primary Frequency Control. 15th European Conference on Multi-Agent Systems (EUMAS), Dec 2017, Évry, France. pp.79-95， 10.1007/978-3-030-01713-2_7 . hal-02330511

\section{HAL Id: hal-02330511 \\ https://hal.science/hal-02330511}

Submitted on 24 Oct 2019

HAL is a multi-disciplinary open access archive for the deposit and dissemination of scientific research documents, whether they are published or not. The documents may come from teaching and research institutions in France or abroad, or from public or private research centers.
L'archive ouverte pluridisciplinaire HAL, est destinée au dépôt et à la diffusion de documents scientifiques de niveau recherche, publiés ou non, émanant des établissements d'enseignement et de recherche français ou étrangers, des laboratoires publics ou privés. 


\title{
Agent-based Security Constrained Optimal Power Flow with primary frequency control
}

\author{
Maxime Velay ${ }^{12}$, Meritxell Vinyals ${ }^{1}$, Yvon Bésanger ${ }^{2}$, and Nicolas Retière ${ }^{2}$ \\ 1 CEA, LIST, Laboratoire d'Analyse des Données et d'Intelligence des Systèmes, \\ Gif-sur-Yvette, 91191 France, \\ maxime.velayecea.fr, \\ ${ }^{2}$ Univ. Grenoble Alpes, CNRS, Grenoble INP ${ }^{\star}$, G2Elab, 38000 Grenoble, France, \\ maxime.velay@g2elab.grenoble-inp.fr
}

\begin{abstract}
We propose in this paper a distributed method to solve the security constrained optimal power flow problem (SCOPF) that considers not only contingencies on transmission lines but also on generators. With this aim, we extend the formulation of the SCOPF problem to consider the primary frequency response of generators as well as the short term constraints of generators and transmission lines. Then, we distribute the problem among different agents and we use a decentralized decision making algorithm, based on the Alternating Direction Method of Multipliers (ADMM), to optimize the grid power supply while being resilient to violations that would occur during contingencies. Finally, we prove the effectiveness of this formulation and implementation on a simple test system.
\end{abstract}

Keywords: Distributed optimization, Multi-Agent System, Security-Constrained Optimal Power Flow, Primary Frequency Control

\section{Introduction}

The planning and operation of power systems is one of the more challenging problems faced by system operators given the complex interplay of multiple objectives to be achieved, including economic, security and reliability aspects. On one side, electricity is a commodity that cannot be easily stored so system operators need to keep the balance between generation and consumption at all times while minimizing the total operation cost of the power system and enforcing the network's operational constraints (e.g. the capacity of the transmission lines). On the other side, transmission system operators also need to perform contingency analysis to guarantee not only that no operational constraint is violated during the normal operating case, but also on potential contingency scenarios when the outage of some components occurs. Most of the transmission system operators (TSOs) must operate at least in compliance with the N-1 criteria so that any single element contingency can be handled and lead to a stable operating point, i.e., with no propagation of the disturbance [10].

Consequently, system operators employ optimization techniques to guarantee that all constraint above are respected as well as to minimize the cost of operation, to solve

\footnotetext{
^ Institute of Engineering Univ. Grenoble Alpes
} 
the so-called Security-Constrained Optimal Power Flow (SC-OPF) [3]. The SC-OPF problem is a fundamental optimization problem in power systems and has been extensively investigated by many researchers.

In current practices, transmission system operators adopt centralized optimization approaches for solving SCOPF problem, which gather all information and make decisions for their own systems. However, during the last decade, power systems have been extended by applying interconnections to the neighboring systems in order to achieve technical and economical advantages, leading to problems of unprecedented scale (e.g. 36 countries interconnected in Europe). As large interconnected power networks come into existence (i.e. covering parts of or even whole continents), such centralized approach raises more and more computation and communication concerns [12].

To avoid these drawbacks, new distributed optimization techniques have been proposed so that the computation can be parallelized (and so it does not increase exponentially with the size of the problem) and control is as much as possible autonomous. Under such approaches, the problem is usually modeled by means of a network of autonomous entities (aka agents) where each entity cooperate by solving a local problem (with local constraints and local data) providing an holistic view for power network operation. Of particular interest here is the Alternating Direction Method of Multipliers (ADMM), a distributed algorithm intended to blend the decomposability of dual ascent with the superior convergence properties of the method of multipliers for constrained optimization [2].

ADMM has been recently applied to a wide variety of a large-scale power system optimization problems. In particular, Kranning et al. in [7], have shown how a decentralized algorithm based on ADMM, can be efficiently applied to the optimal power flow problem (i.e. without considering any contingency scenario) and solved distributively by autonomous agents [11]. More recently, Chakrabarti et al. [4] extended the framework presented in [7] to be able to solve the SCOPF problem. Despite its potential, the model proposed in [4] has an important drawback: it does not take into account the automatic response of generators after a power disturbance and hence it is not able to model any contingency scenario that leads to power imbalance. As a result, such model can not support any contingency involving the lost of a generator and the solution found under such model will never meet the $\mathrm{N}-1$ criteria.

In practice, generators implement primary frequency control (PFC) strategies to steer away the power system from frequency instability. Primary frequency control involves all actions performed locally at the generator to stabilize the system frequency (i.e. within specified stable limits but different from its nominal value) after a power disturbance. Since the power system frequency reflects the power balance and it is the same across the whole power system, the generating units use the frequency to regulate the power supplied (i.e. with a contribution that depends on the frequency deviation and on the generators characteristics).

Against this background, this paper overcomes this drawback by extending the framework in [4] in order to take into account the automatic primary frequency response of generators. By doing so we are able to model contingencies states in SCOPF due to an incident involving a modification of the active power balance and, in particular, those involving generation outages. The major modeling issue is the codependent 
relationship between the control variables (i.e. the output of generators) in the normal operating scenario and the automatic frequency response of generators following the incident. In summary, the SCOPF problem considering the PFC setting is complex, and in this paper we provide the first agent-based totally distributed solution to this challenge.

In more details, this work can be seen as having the following contributions to the state-of-the-art:

- We illustrate the limitations of the SCOPF formulation without PFC by means of a simple numerical example.

- We extend the SCOPF formulation from [4] by: 1) introducing a new variable representing the frequency deviation which is computed by distributed consensus among agents and used to coordinate the power reallocation process after an incident; 2) enhancing the local problem of each generator to consider how it will adjust its production after a contingency following its primary frequency regulation curve.

- We distribute the resulting SCOPF problem among different agents and we use the ADMM algorithm as a coordination mechanism among these agents.

- We evaluate our approach on a IEEE test system to validate its efficiency.

The rest of this paper is structured as follows. A review of the related literature is provided in Section 2. Section 3 gives some background on the decentralised SCOPF formulation and on the ADMM algorithm. Section 4 uses a 3-bus circuit to illustrate the operation and importance of taking into account PFC in SCOPF. Section 5 extend the existing decentralised SCOPF formulation (in particular agent's objective functions and the corresponding ADMM updates) in order to be able to consider PFC. Finally section 6 presents results on the IEEE 14-bus test system and section 7 concludes.

\section{Related work}

The main challenges and techniques for solving the SCOPF are reviewed in [3]. Most of the literature takes into account medium term post-contingency or tertiary frequency control scenarios that correspond to an optimal response of the ISO, like in [8]. Moreover, SCOPF models in the literature are classified into two types: (i) the preventive [2], in which there is no post-contingency re-scheduling of control variables (the solution found for the normal state is also feasible for all contingencies scenarios); and the corrective/curative [9], in which the control variables are allowed to be re-scheduled to rectify any violated operating constraint in post contingency network. The focus of this paper is on short term post-contingency scenarios with automatic reactions, i.e. preventive SCOPF model, of the system that include the primary frequency control as modeled in [6].

Related work on distributed optimization for power system operation can be found in [12] and the references cited therein. Based on the type of information being exchanged, [12] divides the distributed methodologies applied in power system operation into two categories: (i) generator-based decomposition with price/cost information exchange and (ii) geography-based decomposition with physical information exchange. 
On the one hand, generator-based decomposition with price/cost information exchange approaches set each generator as a local control agent. Under this category, a lot of works have been proposed based on different techniques, varying from the incremental cost consensus based methods [13] to the flooding-based consensus approaches [5]. However, by decomposing the central power system operation at generator level, such approaches require significant information exchange at the bus level and hence, they are only efficient when neglecting system-level constraints (e.g. network constraints and capacity limits of transmission lines). Therefore, such approaches are not suitable for solving large-scale OPF problems and even less for solving the extended SCOPF problems (i.e. with system-level security constraints), which is the focus of this paper.

On the other hand, geography-based decomposition methods exchange, instead of cost information of generators, information related to the physical measures (i.e. voltage and power flows). A major advantage of geography-based decomposition approaches is that they divide the large system into several smaller-scale geographical regions coupled by lines and hence they can provide a natural decomposition structure which is consistent with the topology of power physical systems. In this context, ADMM has been identified as one of the most applicable and efficient decomposition methods given its good computational performance and linear convergence rate. ADMM [2] is a distributed solution that combines the fast convergence properties of augmented Lagrangian-based methods with the separability of alternating optimization.

In particular, Kraning et al. proposed in [7] a methodology for decomposing the OPF problem among a collaborative agent network and a fully-distributed ADMMbased OPF algorithm to solve it. The convergence criterion is provided and experiments on large systems are conducted. Chakrabarti et al. [4] extended that model in order to deal with the SCOPF problem, handling different reliability constraints across multiple scenarios. However, they only consider contingencies on transmission lines and hence the primary frequency control is not modeled as the power balance is kept after each contingency. Moreover, the paper lacks empirical evaluation: the framework is only evaluated in a single two bus system.

In summary, to the best of the authors knowledge, the preventive SCOPF including the primary frequency control has never been addressed using a ADMM based distributed algorithm. Hence, this work is the first to propose a decentralized formulation of the preventive SCOPF problem, and a subsequent implementation solved by distributed autonomous agents, that is able to consider contingencies generating power imbalance, and specifically, on generators.

\section{Background}

In this section, we review the ADMM algorithm and its application to the SC-OPF problem. Following the network model proposed by Kraning et al. [7], we divide the set of power system network components into two groups: (i) the set of nets $(N)$, that similarly to the electrical bus concept contains all the loss-less components that connect devices; and (ii) the set of devices $(D)$, that is composed of all power components that are not buses namely transmission lines, generators and loads. These components are the agents of our system. Then, each agent $a \in N \cup D$ (i.e. either device or net) is 
associated to a local objective function $f_{a}\left(x_{a}\right)$ that returns the exploitation cost of agent $a$ for the set of variables $x_{a}$ and a set of constraints, denoted as $C_{a}$, that $x_{a}$ should satisfy in order to be a feasible planning.

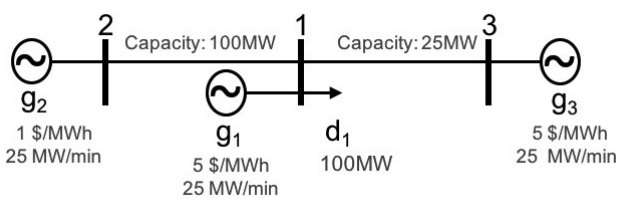

(a) 3-bus circuit

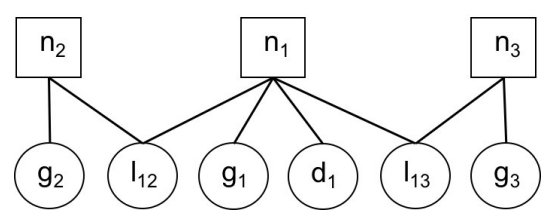

(b) Network model

Fig. 1: A simple bus test circuit (left); its graphical representation in the network model from [7] (right).

In this model, the global objective function is factorized into smaller functions, one for each network agent:

$$
\begin{gathered}
\min _{x} \sum_{d \in D} f_{d}\left(x_{d}\right)+\sum_{n \in N} f_{n}\left(x_{n}\right) \\
\text { subject to } \forall d \in D: x_{d} \in C_{d}, \forall n \in N: x_{n} \in C_{n}
\end{gathered}
$$

Now, we create an edge for every pair of agents whose objective function have some variable in common (i.e. the cost and/or the feasibility of both agents depends on at least some shared variables). We will refer to this set of edges as terminals $(T)$. For each agent $a \in N \cup D$, we use $a$ to refer to both the agent itself as well as to the set of terminals associated with it, i.e., we say $t \in a$ if terminal $t$ is associated with agent $a$. As shown in [7], for a power network this leads to a bipartite graph between nets and devices in which each terminal $t$ connects a device and a net. For example, Fig. 1a shows a simple 3-bus circuit whereas Fig. 1b shows its network model where nets are represented by rectangles, terminals by lines and devices by circles. Moreover, the set of variables associated to terminal $\left(x_{t}=x_{d} \cap x_{n}\right)$ results on the classic power flow variables, namely active power (p) and other quantities that depend on the transmission line and power flow model used. In this paper, we restrict ourselves to DC-model and thus, only the voltage angle $(\theta)$ will be considered for transmission lines and nets, in addition to the active power.

This model is used in [4] to solve a SC-OPF problem in which the optimization is performed over a number of possible contingency scenarios, $\mathcal{L} \in \mathbb{N}^{+}$, each related to a contingency. Here we assume that the first scenario, $(0)$, is the one that stands for the base case (with no contingency). Given a contingency $(c)$ we define $D^{(c)}$ as the set of devices that are disconnected in that scenario.

Thus, in a SC-OPF problem, each terminal $t \in T$ has associated one (active) power schedule over the set of contingencies $\mathcal{L} p_{t}=\left(p_{t}(0), \ldots, p_{t}(\mathcal{L})\right) \in \mathbb{R}^{\mathcal{L}}$. Then, for all $\tau \in[(0), \mathcal{L}], p_{t}(\tau)$ is the (real) power consumed (if $p_{t}(\tau)>0$, otherwise produced) by device $d$ through terminal $t$, for the contingency scenario $\tau$. Similarly, for other 
quantities that are associated with each terminal (such as phase schedules), we use an identical notation to power schedules, i.e., $\theta_{t}=\left(\theta_{t}(0), \ldots, \theta_{t}(\mathcal{L})\right) \in \mathbb{R}^{\mathcal{L}}$.

The set of all power schedules associated with an agent $a \in D \cap N$ (being $a$ either a device or a net) is denoted by $p_{a}=\left\{p_{t} \mid t \in a\right\}$, which we can associate with a $|a| \times \mathcal{L}$ matrix. For other quantities that are associated with each terminal (such as phase schedules), we use an identical notation to power schedules, i.e., $\theta_{a}=\left\{\theta_{t} \mid t \in a\right\}$.

Formally, an energy coordination network models the following optimization problem:

$$
\begin{aligned}
& \min _{p, \theta \in \mathbb{R}^{|T| \times \mathcal{L}}} \sum_{d \in D} f_{d}\left(p_{d}, \theta_{d}\right)+\sum_{n \in N} f_{n}\left(p_{n}, \theta_{n}\right) \\
& \text { subject to } \forall d \in D: p_{d}, \theta_{d} \in C_{d}, \forall n \in N: p_{n}, \theta_{n} \in C_{n}
\end{aligned}
$$

where $p, \theta$ are respectively the set of all terminal power schedules ( $p=\left\{p_{t} \mid t \in T\right\}$ ) and phase schedules $\left(\theta=\left\{\theta_{t} \mid t \in T\right\}\right)$.

Following [7,4], this optimization problem can be solved by a distributed coordination protocol based on the Alternating Direction Method of Multipliers (ADMM) [2]. ADMM is an algorithm that blends the decomposability of dual ascent with the superior convergence properties of the method of multipliers for constrained optimization (i.e. guarantees of achieving convergence without assumptions such as strict convexity for functions $f_{d}$ and $f_{n}$ ). Under ADMM formulation, first, the nets agents objective functions are defined over a duplicated copy of the original variables (i.e. denoted as $\dot{p}, \dot{\theta}$ ) to form the augmented Lagrangian, then the equality constraint $(p=\dot{p}, \theta=\dot{\theta})$ is relaxed via a Lagrange multiplier.

In a nutshell, the ADMM algorithm consists in iteratively applying the following three steps at a given iteration $k+1$ :

The device-minimization step (i.e. parallelized among devices agents):

$$
\begin{aligned}
\left(p_{d}^{k+1}, \theta_{d}^{k+1}\right) & =\arg \min _{p_{d}, \theta_{d} \in C_{d}}\left(f_{d}\left(p_{d}, \theta_{d}\right)+\frac{\rho}{2}\left\|p_{d}-\dot{p}_{d}^{k}+u_{d}^{k}\right\|_{2}^{2}\right. \\
& \left.+\frac{\rho}{2}\left\|\theta_{d}-\dot{\theta}_{d}^{k}+v_{d}^{k}\right\|_{2}^{2}\right), \quad \forall d \in D
\end{aligned}
$$

The net-minimization step (i.e. parallelized among nets agents):

$$
\begin{aligned}
\left(\dot{p}_{n}^{k+1}, \dot{\theta}_{n}^{k+1}\right) & =\arg \min _{\dot{p}_{n}, \dot{\theta}_{n} \in C_{n}}\left(f_{n}\left(\dot{p}_{n}, \dot{\theta}_{n}\right)+\frac{\rho}{2}\left\|p_{n}^{k+1}-\dot{p}_{n}+u_{n}^{k}\right\|_{2}^{2}\right. \\
& \left.+\frac{\rho}{2}\left\|\theta_{n}^{k+1}-\dot{\theta}_{n}+v_{n}^{k}\right\|_{2}^{2}\right), \quad \forall n \in N
\end{aligned}
$$

The (price) scaled dual variables update (i.e. parallelized among nets agents):

$$
\begin{aligned}
& \forall n \in N, u_{n}^{k+1}=u_{n}^{k}+\left(p_{n}^{k+1}-\dot{p}_{n}^{k+1}\right) \\
& \forall n \in N, v_{n}^{k+1}=v_{n}^{k}+\left(\theta_{n}^{k+1}-\dot{\theta}_{n}^{k+1}\right)
\end{aligned}
$$

with iteration index $k$ and some scaling parameter $\rho>0$.

The problem, is by construction, already separated in local sub-problems which allows each agent (either net or device) to solve its sub-problem in parallel and coordinate 
via message-passing through terminals. At each iteration, every device agent computes a minimization step for its local objective function (Eq. 3) with an argument that depends on messages passed to it through its terminals by its neighboring nets agents in the previous iteration $\left(\dot{p}_{n}^{k+1}, \dot{\theta}_{n}^{k+1}, u_{n}^{k+1}\right.$ and $\left.v_{n}^{k+1}\right)$. Similarly, each net agent computes its minimization (Eq. 4) and scaled dual variables steps (Eq. 6) with an argument that depends on messages passed to it through its terminals by its neighboring devices agents in the previous iteration $\left(p_{n}^{k+1}, \theta_{n}^{k+1}\right)$. This is done iteratively until a sufficient consistency is reached at each net.

\section{Considering contingencies involving power imbalance - Primary Frequency Control}

This section first highlights the importance of taking into account the primary frequency control in the SCOPF problem by means of a simple example and second, it provides a formal definition of the primary frequency control scheme in power systems.

\subsection{Motivation}

The model proposed in [4], is the first attempt to use the ADMM to solve a preventive SC-OPF problem. However, this model has a major drawback, it neglects the fact that in reality generator controllers are designed to balance the power in emergency cases by means of the so-called primary frequency control (PFC). Then contingencies on generators cannot be considered or it simply removes the generator from the problem. As a result, the solution found by the SCOPF model formulated in [4] does not meet the $\mathrm{N}-1$ criteria (i.e. it deals with outages of lines but not of generators). To illustrate the limits of the mentioned formulation and to present the preventive SC-OPF with PFC, we take as example the 3-bus circuit depicted in Figure 1a.

\begin{tabular}{|c|c|c|c|c|}
\hline & $g_{1}$ & $g_{2}$ & $g_{3}$ & $\$$ \\
\hline OPF & $0 \mathrm{MW}$ & $100 \mathrm{MW}$ & $0 \mathrm{MW}$ & $100 \$$ \\
\hline SCOPF & $100 \mathrm{MW}$ & $0 \mathrm{MW}$ & $0 \mathrm{MW}$ & $500 \$$ \\
\hline SCOPF with PFC & $50 \mathrm{MW}$ & $50 \mathrm{MW}$ & $0 \mathrm{MW}$ & $300 \$$ \\
\hline
\end{tabular}

(a) Base scenario (pre-contingency) dispatch.

\begin{tabular}{|c|c|c|c|}
\hline \multirow{2}{*}{ Contingency } & \multicolumn{3}{|c|}{ Post-contingency dispatch } \\
\cline { 2 - 4 } & $g_{1}$ & $g_{2}$ & $g_{3}$ \\
\hline$g_{2}$ or line 1-2 & $75 \mathrm{MW}$ & $0 \mathrm{MW}$ & $25 \mathrm{MW}$ \\
\hline$g_{1}$ & $0 \mathrm{MW}$ & $75 \mathrm{MW}$ & $25 \mathrm{MW}$ \\
\hline$g_{3}$ or line $1-3$ & $50 \mathrm{MW}$ & $50 \mathrm{MW}$ & $0 \mathrm{MW}$ \\
\hline
\end{tabular}

(b) SCOPF with PFC post-contingency scenarios dispatch

Table 1: (a) Different models base case solutions and (b) contingency scenarios SCOPF with PFC solutions for the 3-bus circuit in Fig. 1a

Table 1a states the base case solutions for this 3-bus circuit and the different models considered. Observe that the OPF solution (e.g. without considering any security constraint) for this circuit is that the cheapest generator (i.e. $g_{2}$ ) produces all active power 
consumed by $d_{1}$ with a cost of $100 \$ / h$. Now, consider a solution that is not only feasible under normal operating limits, but also after a contingency happened. Notice that in this circuit, any contingency related to the loss of a line also results in the disconnection of a generator (i.e. the loss of line 1-2 disconnects $g_{2}$ and of line 1-3 disconnects $g_{3}$ ). Therefore, the only solution to the SC-OPF problem with no PFC is that generator $g_{1}$ produces all power consumed by $d_{1}$ with a cost of security of $400 \$ / h$. However, notice that this solution is not $\mathrm{N}-1$ resilient since it does not support the loss of generation $g_{1}$ and as we will see next the cost of the security can be lowered.

Now consider the case of SC-OPF with PFC and the contingency on line 1-2. In this case, generators $g_{1}$ and $g_{3}$, both taking part into the PFC, will automatically increase their output to compensate the loss of $g_{2}$ according to their characteristics and droop. Considering that both generators have the same characteristics (e.g. same size and same droop), the units produced by $g_{2}$ will be equally compensated by the remaining generators, namely $g_{1}$ and $g_{3}$. However, the primary response of generators is limited by their ramp rate, and hence generators $g_{1}$ and $g_{3}$ are able to compensate a maximum of $25 \mathrm{MW}$ each. Consequently, in the dispatch, the output of $g_{2}$ should be limited to 50MW whereas the remaining generation is distributed between $g_{1}$ and $g_{3}$. Since the output of generator $g_{3}$ is limited by the maximum capacity of line $1-2$, the only way to avoid the overload of the line is to set the production of $g_{3}$ in the base case to 0 .

Moreover, as summarized in Table $1 \mathrm{~b}$, considering contingencies on line 2-3, and even on any generator, does not add anymore constraint to the problem and hence the solution of the SCOPF when taking into account PFC meets the $\mathrm{N}-1$ criterion. The cost of security is $300-100=200 \$ / h$ when considering the PFC, and represents half the one found by the SC-OPF without PFC.

As a results, taking into account the PFC allows to consider the disconnections of generators and lines that connect generators to the grid. The security of the system is then improved and the N-1 security criteria can be totally enforced.

\subsection{Primary frequency control}

The primary frequency control (PFC) aims at regulating the frequency of the power system by adapting the generation [1]. Since this paper focuses on preventive SC-OPF, the change in generation production variables, following a contingency, is only due to the response of the power system automatic control:

$$
p_{g}^{(c)}=p_{g}^{(0)}+\Delta p_{g}^{(c)}
$$

where $p_{g}^{(c)}$ is the generation after PFC due to contingency $(c)$ and $p_{g}^{(0)}$ is the generation in the base case $(0)$, i.e. prior any contingency.

The primary frequency response follows the following five principles:

1. The active power imbalance due to contingency is completely compensated by the active production of all units taking part to the primary frequency control.

$$
\sum_{g \in G} p_{g}^{(0)}-\sum_{g \in G} p_{g}^{(c)}=0
$$


2. The units taking part to the primary frequency control recover the active power imbalance according to its coefficient: each generator $g$ participating in the PFC responds proportionally to the frequency deviation $\Delta f^{(c)}$ due to contingency $(c)$. $\Delta p_{g}^{(c)}$ is the contribution of the generator to the regulation of the frequency of the system for a deviation of $\Delta f^{(c)}$ on a base frequency of $f_{0}$.

$$
\Delta p_{g}^{(c)}=-K_{g} \cdot \frac{\Delta f^{(c)}}{f_{0}}
$$

where:

$K_{g}$ is the ratio of the nominal active power and the speed droop of the generator (both constants and depending on the generators characteristics)

$f_{0}$ is regulated frequency of the grid $(50 \mathrm{~Hz}$ or $60 \mathrm{~Hz}$ depending of the country)

3. The active production of each generator has to remain within its production limits

$$
P^{\min } \leq p_{g}^{(c)} \leq P^{\max }
$$

4. The primary response of each generator does not exceed the ramp constraints, $\Delta p_{g}^{(c)}$ is limited because generators cannot change their production at any speed.

$$
R^{\min } \leq \Delta p_{g}^{(c)} \leq R^{\max }
$$

5. Once a generator reaches its (ramp or production) limits the other generators have to compensate the non-allocated power according to their own speed droop. Thus, when generators do not change as expected because they reached some constraints, this is reflected into the frequency deviation $\Delta f^{(c)}$ which increases to have the rest of generators compensate more.

For the rest of the paper, we introduce the variable $\alpha^{(c)}$ for the contingency $(c)$ so that:

$$
\alpha^{(c)}=-\frac{\Delta f^{(c)}}{f_{0}}
$$

$\alpha^{(c)}$ is the relative frequency deviation related to contingency $(c)$.

\section{Formulation of nets and devices agents objective functions, constraints and their proximal functions}

In this section, we present the objective functions introduced in Eq. 1, we consider buses and three types of devices, i.e. generators, loads and lines.

\subsection{Nets agents}

Nets are loss-less energy carriers (i.e. buses) with zero cost function but with constraints on the power and phase schedules of their terminals.

A net $n \in N$ requires power balance in each scenario, which is represented by the constraints:

$$
\sum_{t \in n} \dot{p}_{t}^{(c)}=0, \forall(c)=(0), \ldots, \mathcal{L}
$$


In addition to power balance, each net imposes phase consistency via the constraints:

$$
\dot{\theta}_{t}^{(c)}=\dot{\theta}_{t^{\prime}}^{(c)}, \forall t, t^{\prime} \in n, c=(0), \ldots, \mathcal{L}
$$

Thirdly, to consider primary frequency control, each net constrains that in each scenario all the terminals have the same frequency deviation:

$$
\dot{\alpha}_{t}^{(c)}=\dot{\alpha}_{t^{\prime}}^{(c)}, \forall t, t^{\prime} \in n, c=(0), \ldots, \mathcal{L}
$$

Then, the computation of the net-minimization step to calculate the desired values $\dot{p}_{n}, \dot{\theta}_{n}$ and $\dot{\alpha}_{n}$ can be simplified as in [7] ${ }^{3}$ as follows:

$\forall(c) \in \mathcal{L}, \forall t \in n$,

$$
\begin{aligned}
\dot{p}_{t}^{k+1(c)} & =p_{t}^{k+1(c)}-\frac{1}{|n|} \sum_{t \in n} p_{t}^{k+1(c)} \\
\dot{\theta}_{t}^{k+1(c)} & =\frac{1}{|n|} \sum_{t \in n} \theta_{t}^{k+1(c)} \\
\dot{\alpha}_{t}^{k+1(c)} & =\frac{1}{|n|} \sum_{t \in n} \alpha_{t}^{k+1(c)}
\end{aligned}
$$

\subsection{Generators agents}

A generator is a single terminal device which produces power. The local problem of a generator depends on its power production in each case, $p_{g}$, and on a variable that represents the strength of the corresponding steady-state relative frequency deviation for each contingency, $\alpha_{g}$.

Generators have a local cost for operating the generator at a given power level. This cost of operation only accounts for the base case. Indeed, contingencies are not expected to happen in a regular basis so the solution found by the SCOPF is expected to be resilient in front of a contingency but the cost of operation of the generation in such a case is not so important. A quadratic cost function for generating costs:

$$
f_{g}\left(p_{g}^{(0)}\right)=\beta \cdot\left(p_{g}^{(0)}\right)^{2}+\gamma \cdot p_{g}^{(0)}
$$

where $\beta, \gamma>0$ are respectively linear and quadratic cost coefficients.

If the contingency case implies the outage of the generator, the power output of the generator in this case should be zero:

$$
p^{(c)}=0, \quad \forall\left\{(c) \in(1) \ldots \mathcal{L} \mid g \in D^{(c)}\right\}
$$

In the rest of contingencies cases, the primary frequency response of a generator is proportional to its coefficient and bounded by its ramp limits:

\footnotetext{
${ }^{3}$ Eq. 13 is a projection on an hyperplane.
} 


$$
\forall(c) \in\left\{(1) \ldots \mathcal{L} \mid g \notin D^{(c)}\right\} \quad \Delta p_{g}^{(c)}= \begin{cases}R_{g}^{\text {min }} & \text { if } K_{g} \alpha_{g}^{(c)} \leq R_{g}^{\text {min }} \\ K_{g} \alpha_{g}^{(c)} & \text { if } R_{g}^{\text {min }} \leq K_{g} \alpha_{g}^{(c)} \leq R_{g}^{\text {max }} \\ R_{g}^{\text {max }} & \text { if } K_{g} \alpha_{g}^{(c)} \geq R_{g}^{\max }\end{cases}
$$

In each case, the power output of the generator has to remain within its production limits:

$$
\begin{gathered}
\forall(c) \in\left\{(1) \ldots \mathcal{L} \mid g \notin D^{(c)}\right\} p_{g}^{(c)}= \begin{cases}P_{g}^{\min } & \text { if } p_{g}^{(0)}+\Delta p_{g}^{(c)} \leq P_{g}^{\text {min }} \\
p_{g}^{(0)}+\Delta p_{g}^{(c)} & \text { if } P_{g}^{\text {min }} \leq p_{g}^{(0)}+\Delta p_{g}^{(c)} \leq P_{g}^{\text {max }} \\
P_{g}^{\max } & \text { if } p_{g}^{(0)}+\Delta p_{g}^{(c)} \geq P_{g}^{\max }\end{cases} \\
P_{g}^{\text {min }} \leq p_{g}^{(0)} \leq P_{g}^{\max }
\end{gathered}
$$

Unfortunately, the step functions in Eq. 21 and Eq. 22 leads to a non-convex deviceminimization problem. To overcome this, we substitute them by simpler constraints that directly bound the domain of variable $\alpha^{(c)}$ so that $-R_{g}^{\min } \leq K_{g} \alpha^{(c)} \leq R_{g}^{\max }$ and variable $p_{g}^{(c)}$ so that $P_{g}^{\min } \leq p_{g}^{(0)}+\Delta p_{g}^{(c)} \leq P_{g}^{\max }$. Notice that those are more restrictive constraints. In particular, under this assumption when a generator reaches its ramp/production limit, $\alpha^{(c)}$ will not increases and the generators left provide the power that is then missing but instead the base case solution will be modified in order for each generator to contribute to the PFC as planned. This assumption allow us to keep the device-minimization problem for generators convex and hence we can rely on off-the-shelf optimization tools to solve it efficiently.

\subsection{Transmission lines agents}

A (transmission) line is a two-terminal device used to transfer power from one net (i.e. bus) to another. The AC power flow equations are non-convex, so they are often either approximated or relaxed. Here, we use a linear DCOPF model, often used in the literature to get rid of the non-convexity of the physics of AC circuits. Under this model the power flow equations ignore real power losses as well as reactive power and voltage magnitude is assumed to be equal to $1 \mathrm{pu}$. A line has zero cost function but the power flows and voltage phase angles are constrained. In particular, the power flow through the line depends on : (i) the power schedules $\left(p_{l_{1}}\right.$ and $\left.p_{l_{2}}\right)$ and voltage phase angles $\left(\theta_{l_{1}}\right.$ and $\left.\theta_{l_{2}}\right)$ at both sides of the line; and on the susceptance of the line $\left(b_{l}\right)$. In particular, the power and phase schedules should satisfy the relations:

$$
\begin{gathered}
p_{l_{1}}^{(c)}=-p_{l_{2}}^{(c)}=b_{l} \cdot\left(\theta_{l_{2}}^{(c)}-\theta_{l_{1}}^{(c)}\right), \quad \forall(c) \in\left\{(0) \ldots \mathcal{L} \mid g \notin D^{(c)}\right\} \\
p_{l_{1}}^{(c)}=-p_{l_{2}}^{(c)}=0, \quad \forall(c) \in\left\{(0) \ldots \mathcal{L} \mid g \in D^{(c)}\right\}
\end{gathered}
$$

Moreover, each line constrains that in each scenario the power going through the line to be lower than its maximum capacity (i.e. long-term capacity in the base case and 
short-term capacity in a contingency case):

$$
-C_{l}^{\max } \leq p_{l_{1}}^{(c)} \leq C_{l}^{\max }, \forall(c) \in\left\{(0) \ldots \mathcal{L} \mid g \notin D^{(c)}\right\}
$$

Finally, the line also constrains that the steady-state frequency deviation on both sides of the line are equal:

$$
\alpha_{l_{1}}^{(c)}=\alpha_{l_{1}}^{(c)}, \forall(c) \in(0) . . \mathcal{L}
$$

Lines agents proximal problem. To be able to provide a solution we need to change variables to reformulate this problem.

Let's introduce :

$X_{l_{1}}^{(c)}=\left[\begin{array}{c}p_{l_{1}}^{(c)} \\ \theta_{l_{1}}^{(c)}\end{array}\right], Z U_{l_{1}}^{k(c)}=\left[\begin{array}{c}\dot{p}_{l_{1}}^{k(c)}-u_{l_{1}}^{k(c)} \\ \dot{\theta}_{l_{1}}^{k(c)}-v_{l_{1}}^{k(c)}\end{array}\right]$, and $B_{l}=\left[\begin{array}{cc}-1 & 0 \\ \frac{1}{b_{l}} & 1\end{array}\right]$.

$B_{l}$ is a matrix that include the susceptance $b_{l}$ of the line.

We can then write the proximal problems as the minimization of the sum of the augmented Lagrangian terms of each side of the line with the power flow equation and the maximum capacity of the line as constraints. Note that we consider the short-term capacity of lines equals to the long-term capacity for simplicity. The term depending on $\alpha^{(c)}$ is independent.

$$
\begin{aligned}
& \forall(c) \in\{(0) . . \mathcal{L}\} \\
& \underset{X_{l_{1}}^{(c)}, X_{l_{2}}^{(c)}}{\operatorname{minimize}} \quad \frac{\rho}{2}\left\|Z U_{l_{1}}^{k(c)}-X_{l_{1}}^{(c)}\right\|_{2}^{2}+\frac{\rho}{2}\left\|Z U_{l_{2}}^{k(c)}-X_{l_{1}}^{(c)}\right\|_{2}^{2} \\
& \text { subject to } \\
& X_{l_{2}}^{(c)}=B_{l} X_{l_{1}}^{(c)} \\
& {\left[\begin{array}{c}
-P_{l}^{M} \\
-2 \pi
\end{array}\right] \leq X_{i} \leq\left[\begin{array}{c}
P_{l}^{M} \\
+2 \pi
\end{array}\right]}
\end{aligned}
$$

Lines agents proximal problem solution. When the capacity limit is not reached the solution is simply:

$$
\begin{array}{r}
X_{l_{1}}^{(c)}=\left(I+B_{l}^{T} B_{l}\right)^{-1}\left(Z U_{l_{1}}^{k(c)}+B_{l}^{T} Z U_{l_{2}}^{k(c)}\right) \\
\alpha_{l_{1}}=\alpha_{l_{2}}=\frac{\dot{\alpha}_{l_{1}}^{(c)}-w_{l_{1}}^{(c)}+\dot{\alpha}_{l_{2}}^{(c)}-w_{l_{2}}^{(c)}}{2}
\end{array}
$$

When the capacity limits of the line are reached the problem is simplified as the optimal power flow through the line in this case is equal to the maximum capacity.

\subsection{Fixed loads agents}

Therefore, a fixed load is a single terminal device with zero cost function which consists of a desired consumption $l \in \Re$. In this paper we assume that only generation will adapt in front of a contingency (i.e. loads will remain fixed) and hence the solution for a fixed load can be simply summarized as $\forall(c) \in \mathcal{L}, p_{l}^{(c)}=l$. 


\section{Experiments}

The framework described in the previous sections is implemented as a multi-agent system, where agents solve the sub-problems developed in Section 5. The CEA LIST multi-agent system platform based on JADE was used to create those agents and the communication framework.

\begin{tabular}{|c|c|c|c|c|c|c|c|}
\hline & OPF & Base case & Case 1 & Case 2 & Case 3 & Case 4 & Case 5 \\
\hline \hline$\alpha=-\frac{\Delta f}{f_{0}}(\%)$ & - & - & 15.8 & 2.8 & 3.5 & 0.8 & 2.1 \\
\hline \hline Gen. 1 & -168.0 & -138.6 & 0.0 & -156.8 & -161.8 & -144.0 & -152.6 \\
\hline Gen. 2 & -43.3 & -34.5 & -78.6 & 0.0 & -44.2 & -36.7 & -40.4 \\
\hline Gen. 3 & -43.0 & -46.8 & -78.3 & -52.2 & 0.0 & -48.4 & -51.0 \\
\hline Gen. 4 & 0.0 & -10.8 & -42.3 & -16.3 & -17.8 & 0.0 & -15.0 \\
\hline Gen. 5 & -4.7 & -28.3 & -59.8 & -33.7 & -35.3 & -29.9 & 0.0 \\
\hline
\end{tabular}

Table 2: Power generation in MW for each generating unit, comparison between the OPF schedule and the schedule of each case in $\{1,2,3,4,5\}$.

The test system we employed is the IEEE 14-bus test system model available in MatPower. This test is composed of 11 loads, 5 generators with quadratic cost and 20 lines. We modified the model to include ramp constraints of generators and line capacity limits that were missing. In particular, each generator is modelled with a ramp limit of $50 \mathrm{MW}$ and with a speed droop of 5\%. Moreover all lines capacity limits have been set to $110 \mathrm{MW}$ for both, short-term and long-term settings. Regarding ADMM parameters, the scaling parameter was set to $\rho=1$ and the absolute tolerance to $\epsilon=10^{-5}$ for all tested scenarios.

To validate the extension presented in this paper, we restrict our experiments to consider contingencies on generators. Different contingency lists are tested, from a single to all generators. Table 2 presents in detail the case where all single-generator contingencies are considered : case $\{1,2,3,4,5\}$. It provides the power generation of each generator for the base case and in each contingency scenario, compared to the OPF schedule, as in the example of Section 4.1. It also provides the value of $\alpha$ for each contingency, for example, if the generator 1 is disconnected the steady-state frequency deviation on a $50 \mathrm{~Hz}$ system would be equal to $7.9 \mathrm{~Hz}$. The assumption in Section 5.2 that constrains the relative frequency deviation $(\alpha)$ can be justified in this example. Not constraining $\alpha$ would result in a cheaper solution but at the cost of a deeper frequency deviation and most of $50 \mathrm{~Hz}$ generators would have difficulties to handle a frequency deviation of $7.9 \mathrm{~Hz}$, and so potentially even more if the frequency deviation is greater.

Figure 2 compares the generation cost of our SCOPF solution with respect to those of the OPF solution to illustrate the cost of security. The different contingency lists are then sorted from the cheapest to the more expensive. Notice that the considerations of generators 2 and 3 have the greatest impact on the cost of security even though these generators disconnection imply a relatively small frequency deviation. It thus justifies 

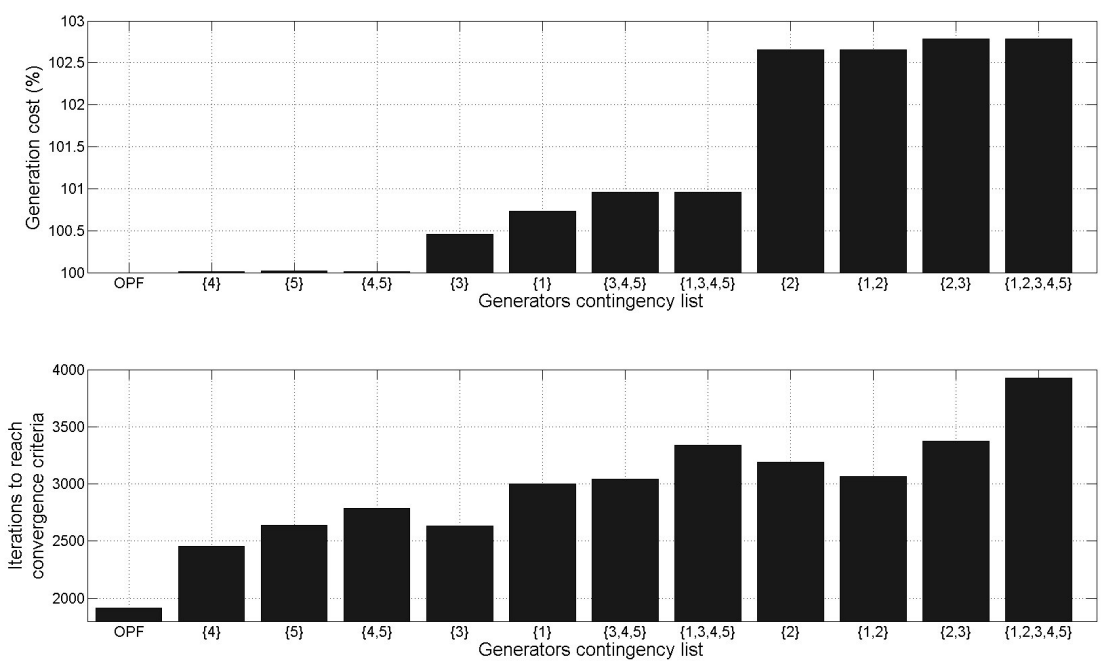

Fig. 2: Comparison of the SCOPF with different contingency lists with the OPF. Number of iterations needed to converge and the cost of generation in percentage of OPF cost.

the need of considering the more contingencies possible, and so deal with large number of contingency scenarios, even when the contribution of the devices do not seem significant compare to others, like generator 1 .

We also compare the number of iterations needed to reach the convergence criteria and this comparison highlights the strength of this type of distributed method. In particular, observe that there are 5 control variables for generators in the OPF and 14 for the bus angles and for contingency cases $\{1,2,3,4,5\}$ there are 5 more for the frequency deviations and 5 times 14 bus angles to determine with different constraints considered in each case and between cases. We notice here that the number of iterations needed to converge did not increase as much as the complexity of the problem solved. This result is promising because it proves a good scalability of the method to the number of contingency scenarios.

\section{Conclusions and future work}

We extend a previous decentralized security-constrained optimal power flow framework to take into account the automatic primary frequency control of generators and we solve it in a fully distributed way using a ADMM-based algorithm. The contribution of this paper allows this distributed SCOPF model to find solutions that remain stable after the disconnection of generators in the system. We have also presented a multi-agent implementation of the method in which individual local agents are restricted to access their own data and exchange relevant information with their neighbors following ADMM 
iterative equations. To evaluate the efficiency of our approach we provide results on the IEEE 14-bus test system. Empirical results show how our method is able to find optimal SCOPF solutions for this circuit, defining for each contingency case the corresponding power flows and steady-state frequency deviation.

In future work, we plan to design a benchmark to be able to validate our approach on larger power system networks and quantify its performance, in particular regarding its scalability. We also plan to test the approach using more complex device models, e.g. the non convexity brought by generators' ramp constraints. The ability of our approach to solve the resulting more complex problem should then be tested extensively.

\section{References}

1. P. Bornard and M. Pavard. Réseaux d'interconnexion et de transport: réglages et stabilité. Technical report, Téchniques de l'ingénieur, 1993.

2. S. Boyd, N. Parikh, E. Chu, B. Peleato, and J. Eckstein. Distributed Optimization and Statistical Learning via the Alternating Direction Method of Multipliers. Foundations and Trends in Machine Learning, 3(1):1-122, 2011.

3. F. Capitanescu, J. M. Ramos, P. Panciatici, D. Kirschen, A. M. Marcolini, L. Platbrood, and L. Wehenkel. State-of-the-art, challenges, and future trends in security constrained optimal power flow. Electric Power Systems Research, 81(8):1731-1741, 2011.

4. S. Chakrabarti, M. Kraning, E. Chu, R. Baldick, and S. Boyd. Security Constrained Optimal Power Flow via proximal message passing. 2014 Clemson University Power Systems Conference, pages 1-8, 2014.

5. W. T. Elsayed and E. F. El-Saadany. A fully decentralized approach for solving the economic dispatch problem. IEEE Transactions on Power Systems, 30(4):2179-2189, July 2015.

6. K. Karoui, H. Crisciu, and L. Platbrood. Modeling the primary reserve allocation in preventive and curative security constrained opf. In Transmission and Distribution Conference and Exposition, 2010 IEEE PES, pages 1-6. IEEE, 2010.

7. M. Kraning, E. Chu, J. Lavaei, and S. P. Boyd. Dynamic network energy management via proximal message passing. Foundations and Trends in Optimization, 1(2):73-126, 2014.

8. A. Monticelli, M. V. F. Pereira, and S. Granville. Security-constrained optimal power flow with post-contigency corrective rescheduling. IEEE Transactions on Power Systems, 2(1):175-180, 1987.

9. D. T. Phan and X. A. Sun. Minimal impact corrective actions in security-constrained optimal power flow via sparsity regularization. IEEE Transactions on Power Systems, 30(4):19471956, 2015.

10. A. Pinar, J. Meza, V. Donde, and B. Lesieutre. Optimization strategies for the vulnerability analysis of the electric power grid. SIAM Journal on Optimization, 20(4):1786-1810, 2010.

11. P. Scott and S. Thiébaux. Distributed multi-period optimal power flow for demand response in microgrids. In Proceedings of the 2015 ACM Sixth International Conference on Future Energy Systems, e-Energy 2015, Bangalore, India, July 14-17, 2015, pages 17-26, 2015.

12. Y. Wang, S. Wang, and L. Wu. Distributed optimization approaches for emerging power systems operation : A review. Electric Power Systems Research, 144:127-135, 2017.

13. Z. Zhang and M.-Y. Chow. Convergence analysis of the incremental cost consensus algorithm under different communication network topologies in a smart grid. IEEE Transactions on power systems, 27:1761-1768, 2012. 